measures included analysis of handover duration and a postimplementation staff survey to investigate staff perception and experience.

Results There is a sustained reduction in the length of the post-ward round handover from 94 to $35 \mathrm{~min}$ (10 days analysed pre and post-intervention). A qualitative survey found the following percentages of people agreed or strongly agreed that: job allocation is more robust and efficient (93\%), handover is more efficient (64\%) and there is improved patient 'ownership' (86\%). 93\% of respondents agreed that an active 'pause' at the end of the handover to ask questions or discuss staff and service issues was beneficial. The response rate was 14 out of 33 possible respondents (42\%). A key finding was that the respondents unanimously agreed that facilitating a junior-led handover was empowering for trainees and turned a service safety task into a training opportunity.

Conclusion Empowering junior members of the team to hand over patients in conjunction with a series of small changes improved the effectiveness and efficiency of the medical handover. It also enabled a cultural change as handover was seen as a training opportunity in addition to being a service function.

\section{G390(P) HOSPITAL AT NIGHT: OUR EXPERIENCES IN IMPROVING PATIENT CARE THROUGH ESTABLISHING A HAN TEAM}

M Lister, F Osborne, S Joseph. Acute Receiving Unit, Royal Hospital for Sick Children, Edinburgh, UK

\subsection{6/archdischild-2018-rcpch.379}

\section{Aims}

- To set up a Hospital At Night (HAN) team and provide the facilities for effective working.

- To improve communication within the HAN team.

- To reduce interruptions to handovers and maintain handovers within the allocated timeframe.

Background The decision to establish a HAN team was taken following review of the medical cover of a 130 bedded quaternary paediatric hospital. Key issues were:

- A lack of effective team working and inadequate communication leading to workload imbalances and risk of important tasks being missed.

- A poor escalation policy leading to inappropriate escalation of deteriorating patients and patient safety issues.

Methods To address the issues we set up a multidisciplinary working group comprising: a consultant paediatrician, a paediatric registrar, a nurse manager and a nurse co-ordinator. These individuals underwent a peer consultation programme and the working group then met with other HAN teams throughout the region to learn from their experiences. Several audits and 'time in motion' studies identified specific issues which could be addressed. 48 Plan Do Study Act (PDSA) cycles were undertaken over an 18 month period with close collaboration and communication between our project group, senior clinical management and front line teams.

Results Funding was obtained for a specifically adapted 'HAN Room' which has become the team base for handovers, administrative work and breaks. Improvements within this room included: improved IT facilities, lockers, basic catering facilities and increased space. An updated escalation policy for deteriorating patients has been implemented complemented with ward level teaching on identifying and managing a deteriorating patient for nursing teams. Interruptions to handovers have stopped and now 95\% of handovers occur within the allotted time. Variation from this occurs appropriately due to discussion about complex or deteriorating patients.

Conclusion We have set up a successful HAN team and provided it with appropriate facilities. By doing this we have significantly improved communication, handovers, team dynamics and the team culture. The hospital co-ordinator working within the HAN team now provides an excellent link between the HAN team and the ward nurses.

\section{G391(P) SCHOOL-BASED DIABETES CLINICS: QI TO ENGAGE FREQUENT NON-ATTENDERS AND IMPROVE TEENAGER'S SELF-MANAGEMENT}

\begin{abstract}
${ }^{1,2}{ }^{2}$ Alins Sahun, ${ }^{2} \mathrm{~K}$ Camara, ${ }^{2,3} \mathrm{~K}$ Gething, ${ }^{2} \mathrm{~J}$ Gane, ${ }^{2} \mathrm{D}$ Schenck, ${ }^{4,5} \mathrm{Y}$ Tse. ${ }^{1}$ Paediatric Diabetes Team, Plymouth Hospitals NHS Trust, Plymouth, UK; ${ }^{2}$ Paediatric Diabetes Team, The Great North Children's Hospital, Newcastle upon Tyne, UK; ${ }^{3}$ Clinical Psychology, The Great North Children's Hospital, Newcastle upon Tyne, UK; ${ }^{4}$ Quality Improvement Service, The Great North Children's Hospital, Newcastle upon Tyne, UK; ${ }^{5}$ Paediatric Renal Team, The Great North Children's Hospital, Newcastle upon Tyne, UK
\end{abstract}

\subsection{6/archdischild-2018-rcpch.380}

Background Optimising attendance rates in outpatient's clinics is important to ensure good clinical care and to avoid waste of scarce medical resources. In 2016, we identified a cohort of 17 teenagers that frequently Do Not Attend (DNA) the Paediatric Diabetes Multidisciplinary Clinics, compromising patient care and reducing compliance with NICE and BPT standards. As this cohort was found to be enrolled in a cluster of 5 schools we explored the benefit of taking diabetes clinics into school.

Objectives Design and deliver additional school-based clinics to facilitate teenager's engagement with healthcare services.

Patients and methods During 6 months quarterly school-based clinics were offered to all teenagers with T1DM in these secondary schools. A semi-structured interview was administered to identify their concerns about diabetes. Patients were invited to provide feedback about hospital clinics. 34 teenagers were targeted (17 initially identified as frequent non-attenders and 17 controls at same schools).

Results The hospital DNA rate was reduced by 50\%. HbA1c levels remained unchanged over the study period but school clinics allowed early identification of patients with rapid deterioration.School-based clinics improved teenagers' ability to express their real beliefs about diabetes and be actively involved in the management of their condition. This unveiled significant knowledge gaps not previously identified by the team in $50 \%$ of the participants. This clinic format improved communication and partnership with school nurses/health assistants, enabling healthcare plans to be more effectively carried out.Although patients were usually happy with hospital-based clinics, they complained about missing school, time spent in hospital and lack of privacy. Some teenagers perceived judgemental attitudes in healthcare professionals or parents.

Conclusions

- This pilot shows promise in improving contact in this difficult to reach group while reducing wasted hospital clinic slots.

- School clinics are an effective way to engage young people in self-management, actively preparing them for transition.

- A well powered trial may allow HbA1c improvement to be shown. 
G392(P) INTRAVENOUS ANTIBIOTIC USE FOR SUSPECTED EARLYONSET BACTERIAL INFECTION IN HEALTHY NEONATES: A SINGLE-CENTRE CLOSED LOOP AUDIT

${ }^{1}$ ER Osen, ${ }^{2}$ GS Krishna. ${ }^{1}$ Ear Nose and Throat (ENT), Colchester Hospital University NHS Foundation Trust, Colchester, UK; '2Department of Paediatrics, Addenbrooke's Hospital, Cambridge, UK

\subsection{6/archdischild-2018-rcpch.381}

Aims We aimed to identify and reduce the number of healthy neonates started on intravenous antibiotics for suspected earlyonset bacterial infection in a district general hospital, and ensure that practice was commensurate with that set out in the relevant NICE (National Institute for Health and Care Excellence) guidance (CG149).

Methods A retrospective audit was performed using the clinical notes, drug charts and pathology reports of neonates who had been started on antibiotics in two 3 week periods, before and after intervention. The intervention was multifaceted and included the introduction of a neonatal sepsis pro forma and decision aid, $(\mathrm{P}) \mathrm{s}$ in relevant clinical areas, and teaching for junior and senior paediatricians.

Results The number of neonates commenced on antibiotics for suspected infection fell by $43 \%$ from 42 to 24 neonates, and compliance with absolute NICE criteria for commencement of antibiotics improved from $69 \%$ to $93 \%$. $76 \%$ of neonates in cycle 1 met NICE guidance for consideration of cessation of antibiotics at 36 hours but $77 \%$ of these neonates received more than 48 hours of antibiotics; similarly, in cycle 2, 73\% of neonates warranted consideration of cessation of antibiotics at 36 hours, but $91 \%$ of these received more than 48 hours of antibiotic treatment in total. In $77 \%$ of cases in cycle $1 \%$ and $82 \%$ of cases in cycle 2 , the documented rationale for continuing antibiotics past 36 hours in these healthy neonates was that blood culture results had not yet been reported.

Conclusion Our intervention was effective in reducing the number of healthy neonates started on intravenous antibiotics and improving compliance with NICE guidance for commencement of antibiotics. The proportion of neonates meriting a short duration of antibiotics was high despite improvement in following approved criteria, suggesting that there may be room for these criteria to be revised to capture fewer healthy neonates. However, our intervention was not successful in reducing duration of antibiotic use in healthy neonates. Establishing processes to accelerate pathology reporting could be the focus of further interventions to improve compliance with national guidelines and minimise unnecessary antibiotic exposure to healthy neonates.

\section{G393(P) JUST A 'SPOON FULL OF SUGAR' HELPS THE BREASTFEEDING RATES GO UP}

CP Darby. Neonatal, Southern Health and Social Care Trust, Craigavon, UK

\subsection{6/archdischild-2018-rcpch.382}

Aim A quality improvement initiative to improve the breastfeeding rates in the Trust to impact on the low breastfeeding rates in Northern ireland.

Method Northern Ireland currently has the lowest breastfeeding rates in the UK. Only $64 \%$ of mothers begin breastfeeding here, compared to $83 \%$ in England, $74 \%$ in Scotland and $71 \%$ in Wales. Mothers in Northern Ireland, who choose to breastfeed, also do so for a shorter period than breastfeeding mothers elsewhere in the UK. As a quality improvement (QI) strategy the areas of point of care that could be improved in order to advocate breastfeeding and optimise not only uptake but also the longevity of breastfeeding at point of discharge from hospital were analysed. The focus of the QI was to change the neonatal hypoglycaemia protocol to eliminate the use of milk formula as a substitute for breastmilk for infants with transient hypoglycaemia. Through critical analysis of the evidence the Trust guideline was replaced with a guideline that replace milk formula with $40 \%$ Glucose gel in combination with breastmilk/breastfeeding to improve neonatal hypoglycaemia management.

Results With the removal of milk formula as the option to treat neonatal hypoglycaemia, mothers were not made to feel inadequate when trying to establish breastfeeding. The use of the $40 \%$ glucose gel and its' ease of use and administration has been well received by midwives, doctors and parents. Breastfeeding rates have increased at the point of discharge from hospital. There has been significant savings within the Trust due to the reduction in milk formula consumption. Parents reported feelings of being 'supported' and 'empowered' when commencing and continuing breastfeeding. A reduction in neonatal admission for neonatal hypoglycaemia was apparent as a secondary outcome due to the change in guideline and intervention.

Conclusion Glucose gel use in combination with breastfeeding improves the support provided to breastfeeding mothers, leading to an increase of breastfeeding rates at the point of discharge from hospital. The successful quality improvement intervention has seen an improvement across two health directorates, Women and Child Health and Paediatrics. There was a reduction in neonatal admissions for neonatal hypoglycemia with improvement outcomes for infants as breastfeeding rates improve.

\section{G394(P) LEARNING FROM PICU TRANSFERS FROM A PAEDIATRIC EMERGENCY DEPARTMENT AND PAEDIATRIC WARD}

S Cuevas-Asturias, S Grossman, T Maggs, J Runnacles, L Sinitsky. Paediatrics and Child Health, Royal Free London NHS Foundation Trust, London, UK

\subsection{6/archdischild-2018-rcpch.383}

Aims Local hospital Paediatricians often rely on updates from retrieval services for information about their patients transferred to Paediatric intensive care units (PICU). This quality improvement project aimed to increase local hospital knowledge about their PICU transfers and identify areas for improvement to enhance patient safety and clinical care.

Methods In November 2016 a new incident log was implemented to collect data on PICU transfers from the Emergency Department and Paediatric ward of one district general hospital. Between January and September 2017, a multi-disciplinary team met monthly to review the Electronic Document and Records Management (EDRM) system of all patients transferred to PICU in the previous month(s). The multidisciplinary team included medical and nursing representation from Paediatric, Emergency and Anaesthetic departments and the outreach resuscitation team. EDRM was reviewed using an adapted RECALL (Rapid Evaluation Cardiorespiratory Arrest with Lessons for Learning) tool. The RECALL tool provided a structured template for retrospective case note review of 
patient management, including monitoring, escalation and medical reviews, in addition to staffing issues, debriefs and parental feedback. Areas for improvement were shared across services by monthly newsletters and updates at teaching sessions and departmental risk meetings.

Results Between November 2016 and September 2017, 26 children aged 2 days to 15 years were transferred to PICU, of which 69\% were from the Emergency Department. The primary problem was respiratory failure in $50 \%$ of cases.

In the first 6 months many cases had incomplete Paediatric Early Warning Score (PEWS) monitoring and/or poor escalation of care documentation. Shared learning and teaching on PEWS resulted in recent cases of exemplary practice. Initially EDRM was incomplete for patients moved to theatre from the emergency department for intubation. Recommendation to scan notes prior to moving resulted in complete EDRM records in all subsequent reviews. The multidisciplinary team recognised that parental views remained unheard and so initiated parents being offered the Friends and Family feedback card post-PICU repatriation.

Conclusion Shared learning from regular structured multidisciplinary case note reviews of PICU transfers results in improvements in the management, safety and documentation of children presenting with critical illness.

\section{G395(P) AN INNOVATIVE APP DESIGNED TO REDUCE HEALTHCARE-RELATED ANXIETY IN YOUNG CHILDREN}

${ }^{1} \mathrm{R}$ Duncombe, ${ }^{2} \mathrm{R}$ Evans Fry. 'Paediatrics, Oxford University Hospitals NHS Trust, Oxford, UK; ${ }^{2}$ Paediatrics, Bucks Healthcare NHS Trust, Aylesbury, UK

\subsection{6/archdischild-2018-rcpch.384}

Aims To create an interactive App that reduces healthcare associated anxiety in young children through education and play.

Method A significant number of children have contact with healthcare in the UK every year, with 3 million children attending $\mathrm{A}$ and $\mathrm{E}$, and 1 in 10-15 children being admitted to hospital. The literature suggests that $20 \%$ of children admitted to hospital suffer some form of emotional or behavioural disturbance. Play is key to childhood development and learning, and is practiced across healthcare settings. Psychological preparation for healthcare experiences has been shown to help reduce patient and parental anxiety. With ever-increasing budget restrictions within the NHS, innovative ideas are required to tackle this problem alongside invaluable play therapy resources.

We created an interactive App for children aged 4-8 years designed to ameliorate distress and improve paediatric healthcare experiences. It familiarises children with hospital environments and the common scenarios they may encounter through interactive stories, games and animations.

A patient and carer survey was undertaken following Phase 1 development of the app (a single character story with animations and games). Twenty children aged 4-8 years were selected at random to play on the App (for up to $20 \mathrm{~min}$ ) with carer supervision. Following this standardised questionnaires were completed by the child (visual rating scales) and carer to assess educational value and usability.
Results The overall feedback from study participants was good with $100 \%$ reporting that they would download the final product and 95\% believing that the completed App would be useful for educating children about hospitals.

Since the release of the App there has been 350 downloads within the first two weeks and positive feedback received via App ratings, social media messages and emails.

Conclusions New and innovative ideas are imperative to develop NHS services for the future. This is an illustration of how digital technology using play, can help to improve Paediatric healthcare experiences and potentially increase the efficiency of a service. A larger study of the beneficial effects of this App is planned following its wider distribution within the NHS.

\section{G396(P) NEONATAL 'VIRTUAL' CLINIC: A QUALITIY IMPROVEMENT PROJECT}

L Fullerton, C Cane. Neonatal Department, Royal Free NHS Foundation Trust, Barnet Hospital, London, UK

\subsection{6/archdischild-2018-rcpch.385}

Aims To improve the follow up of outstanding jobs from the neonatal unit and post-natal ward. Outstanding jobs were often being left for prolonged periods with no dedicated person to follow up and with no record of jobs having been completed. This resulted in poor patient care, inadequate communiation and potential for clinical risk.

Methods A quality improvement project was initiated and a plan, study, do, act cycle was used. Initial review showed that oustanding jobs were put onto the bottom of the clinical handover list and random junior doctors were irreguarly asked to review it during their clinical time. Some jobs had been present for more than a 3 month period with most jobs having inadequate information detailing reasons required for follow up. A number of jobs were inappropriate for junior doctor follow up thereby identifying a lack of education and under utilisation of pathways already in place. We set up a weekly neonatal 'virtual' clinic and amended the rota in conjunction with management and previously allocated admin time for the registrars. Aims and rules of the list were created and the junior doctors were educated as to the changes. A registrar was assigned during their admin time to run a 'virtual' phone clinic, once a week, where they would chase results and contact parents and/or other health professioanls whilst documenting their actions on electronic patient records. Results After a 12 month period, there were significantly fewer outstanding jobs with no jobs waiting longer than 3 months and all jobs deemed appropriate for 'virutal' clinic follow up. There were no clinical risk incidents. Families have informally expressed their satisfaction with having direct hospital contact and promises made as an inpatient fulfilled. This implementation has become a permanent change wihtin the neonatal department.

Conclusion This quality improvement initiative shows that patients can be safely followed up 'virtually.' Communication and documentation between health professionals needs to be sound and of high quality. This can improve the quality of care delivered and improve satisfaction amongst our patients and families. 